\title{
The Effect of Prenatal Stress on Auditory Brainstem Responses in Rat
}

\section{Pups}

\author{
Ebrahim Pirasteh ${ }^{1,2}$, Mehdi Akbari $^{3,{ }^{*}}$, Akram Pourbakht $^{3}$, Amir Hosseini $^{4}$, Mohsen Bahadorifar ${ }^{5}$, \\ Milad Saliminezhad ${ }^{5}$ and Mehdi Mohammadi ${ }^{6}$ \\ ${ }^{1}$ Department of Audiology, School of Rehabilitation Science, Iran University of Medical Sciences, Tehran, Iran \\ ${ }^{2}$ Department of Audiology, School of Rehabilitation, Zahedan University of Medical Sciences, Zahedan, Iran \\ ${ }^{3}$ Department of Audiology, School of Rehabilitation Sciences, Iran University of Medical Sciences, Tehran, Iran \\ ${ }^{4}$ Department of Anatomy and Embryology, School of Medicine, Iran University of Medical Sciences, Tehran, Iran \\ ${ }^{5}$ Department of Audiology, School of Rehabilitation, Shahid Beheshti University of Medical Sciences, Tehran, Iran \\ ${ }^{6}$ Health Promotion Research Center, Zahedan University of Medical Sciences, Zahedan, Iran \\ "Corresponding author: Department of Audiology, School of Rehabilitation Sciences, Iran University of Medical Sciences, Tehran, Iran. Email: akbari.me@iums.ac.ir
}

Received 2017 June 17; Revised 2017 August 14; Accepted 2017 August 21.

\begin{abstract}
Background: Recent epidemiological evidence indicates that the fetal environment can influence susceptibility to later disease during the lifespan. Prenatal stress exposure alters the programming of the metabolic and endocrine balance of various organs, including the auditory system.

Objectives: The current study aimed at evaluating how prenatal stress influences Auditory Brainstem Responses.

Methods: Eighteen pregnant Wistar rats were stressed during a one-month gestation by chronic mild stress (CMS, a variable schedule of different stressors). After birth, the offspring's hearing thresholds and latencies were evaluated and compared with the control group. The hearing thresholds were assessed by recording auditory evoked brainstem responses to 4, 8,12, and $16 \mathrm{kHz}$ tone burst. Results: The results showed that although CMS caused a significant increase in corticosterone in pregnant mothers, the offspring with CMS experience had significantly lower body weight than control animals $(\mathrm{P}<0.00)$ yet there was no difference between hearing sensitivity of prenatally stressed offspring and the control group. However, ABRs showed a marked prolongation in wave II and IV latencies in prenatally stressed rats $(\mathrm{P}<0.05)$.

Conclusion: The current data confirmed that prenatal exposure to mild stress is not detrimental to hearing sensitivity. However, these data suggest that prenatal stress can affect the temporal processing of auditory stimuli in the brainstem.
\end{abstract}

Keywords: Prenatal Stress, Hearing System, Auditory Brainstem Response, Rat

\section{Background}

Results from recent animal studies have implied that offspring of mothers, who have been under stress during their pregnancy may be at risk of developing various physical and mental problems (1). Today, stress as one of the most significant health problems in many communities, has been studied extensively. One of the most important causes of stress are environmental and occupational problems, especially in pregnant females. It has been proven that stress has a direct impact on the health of the fetus (2). One way to investigate stress, which is often used to study rodent's different pathologies, is prenatal stress (2). To create such stress patterns, a chronic mild stress (CMS) regimen is usually used (2). Following introduction of stress, the level of stress hormones, such as glucocorticoid, is increased in the body (3). Glucocorticoids effectively cross the placenta and affect the fetus (1). Although glucocorticoids play a vital role in differentiation of several tissues, including lungs and heart (4), long-term exposure of the fetal to this hormone can inhibit the growth of the fetus and change its maturity trajectory (1). Since the fetus is passing through its golden developmental stage (3), such hormonal abnormalities could make some basic changes in cell metabolism pattern (3). According to previous studies, animals with a history of prenatal stress show abnormalities in their hypothalamic pituitary adrenal axis (HPA), which is recognized through a number of signs, including raise in basic levels of glucocorticoids as well as long-term upregulation of this hormone after exposure to an acute stressful event (5). Many of deficiencies a person experiences during their lifetime can be related to disruption of the HPA axis (6).

Several studies have reported that prenatal stress affects the auditory system $(1,6)$. Exposure to high levels of glucocorticoids during the fetal period results in differentiation of inner ear tissues with fewer blood vessels and progenitor cells (6). This in turn will damage the cochlea 
(6) and increase in threshold of hearing (5). Furthermore, if these animals are affected by ototoxic agents, they will be harmed even more (7). Animals with a history of stress over the fetal period also show greater involvement of the afferent auditory pathways (7). Of other consequences of fetal stress, impaired sensory gating (8), severe startle reflex (9), dendritic atrophy of auditory cortex (10), atrophy of auditory neurons in IC (11), and neuronal morphology defects in different parts of the central auditory nervous system (CANS) (11) can be mentioned. It was also reported that stress can impair cochlear function on the molecular level (11), which results in sudden hearing loss (SHL) (12), tinnitus and dizziness (13), and hyperacusis (11). However, the effect of exposure to high levels of stress during the fetal period or high levels of synthetic glucocorticoids during pregnancy on cochlea and hearing threshold has not been confirmed in all studies $(1,7)$ and more researches are needed in this field.

One of the major hazards of stress during pregnancy is fetal developmental delay (3). Through blocking growth factors, such as insulin-like growth factor 1 (IGF-1) (14) and increased activity of the HPA axis (5), stress changes the patterns of gene expression and causes the body to produce and store energy materials and increase cardiovascular activities, which often reduce fetal weight gain (14). Furthermore, IGF-1 is a powerful mitogen so when it is reduced, body's ability to maximize the speed of cell cycle during development is disrupted, which is followed by lower number of auditory sensory cells and auditory neurons (10). This issue has increased the prevalence of sensorineural hearing loss (SNHL) in people with lower birth weight (14). These findings have been reported in both animal and human studies $(1,14)$. Therefore, it seems that a person's overall health throughout life is strongly influenced by exposure to high levels of stress during the fetal period (3).

The aim of this study was to evaluate a theory that suggests that auditory brainstem response (ABR) is affected by stress during pregnancy.

\section{Methods}

Eighteen adult virgin female Wistar rats, weighing 200 to $250 \mathrm{~g}$, were purchased from the Center of Experimental and Comparative Studies of Iran University of Medical Sciences. On average, a humidity of $50 \%$ and temperature of $22^{\circ} \mathrm{C}$ to $24^{\circ} \mathrm{C}$ was set for the place where rats were kept with a 12/12 light cycle. Rats were placed together in large standard cages with a 2:1 female to male ratio. While kept in specific cages, the rats had free access to food and water. The presence of a vaginal plug the next morning indicates that mating has occurred and was considered as the first day of pregnancy. Immediately after confirmation of pregnancy, female rats were separated and transferred to other standard cages (one rat per cage). Then, pregnant rats were divided to two equal groups of nine, control and exposed prenatal stress group. Gestational stress was presented at gestation days 9 to 19 in the form of CMS. Stress was randomly imposed on rats using one, two or three stressors in 24 hours so total time of exposure to stress ranged between one to 24 hours a day (Figure 1). Each stressor was presented once or twice, for example the animals were placed in smaller cages $(25 \times 20 \times 14 \mathrm{~cm})$ on the ninth (from 9 am to $4 \mathrm{pm}$ ) and 14th (from 5 am to $10 \mathrm{pm}$ ) day of pregnancy. The stress exposure program in this study was derived from Willner's CMS model (2), whose functionality has been proved in previous studies (1).

Following the CMS protocol presented on the 20th day of pregnancy, tail blood sample were taken by lancing the tail of the rat and collecting a few drops of blood. To minimize variance due to fluctuation of corticosterone during the day, blood samples were collected between 9:00 and 11:00 a.m. Blood plasma separation from cells was done by spinning $3000 \mathrm{rpm}$ at $4^{\circ} \mathrm{C}$. The plasma was stored in a $-20^{\circ} \mathrm{C}$ freezer for corticosterone measurement. Plasma corticosterone concentration was determined by enzyme linked immunosorbent assay (ELISA) technique.

In this study, it was attempted to control other potential stressors as much as possible and provide a normal living environment for the animals. To reduce the stress as a result of separation from the mother, for instance during breastfeeding, the gradual weaning method (Cook, 1999) was used (15). To this end, infants were separated from mothers from day 21 to 27 after birth for a couple of hours each time and kept in new cages. Infants with the same gender and from the same family were placed in these new cages. The cages had the same size and dimensions as that of the mothers. The bedding of the cages was partly new and partly taken from mothers' bedding. It was also attempted to keep animals from different groups in similar conditions both in terms of cage and relevant personnel. Besides, all cages were cleaned twice a week and new bedding was replaced with the old ones.

Male rat offspring were weighed at 27 days old and then divided to two groups, based on exposure to prenatal stress (with and without prenatal stress exposure experience). To control the litter effect factor and match all groups, only one baby of each mother was allocated to one of these groups. The baby rats that had not been placed in these two groups were kept for the next phase of the study.

The ABR was recorded from the right ear via a needle electrode on the vertex, referred to behind the ear. The response was amplified (100 K), filtered (100 to $3000 \mathrm{~Hz})$, and averaged with A - D board in the NATUS acquisition 


\begin{tabular}{|c|c|c|c|c|c|c|c|c|c|c|c|}
\hline Gestation Day & 9 & 10 & 11 & 12 & 13 & 14 & 15 & 16 & 17 & 18 & 19 \\
\hline Smaller Cage & - & & & & & & & & & & \\
\hline Wire Cage & & & & & & & & & - & & \\
\hline No Food/Water & & & - & & & & - & & & & \\
\hline No Water & & & & & & & & & & & \\
\hline Damp Bedding & & & & & & & & & & & \\
\hline Cage Tilting & & & & - & & & & & & & \\
\hline Crowding & & & & & 一 & & & & & & - \\
\hline Alone in Cage & & & & & & & & & & & \\
\hline New Partner & & & & & & & & & & & \\
\hline
\end{tabular}

Figure 1. Gestational stress program that was presented at gestation days 9 to 19 . Vertical borders between gestation days correspond to 00:00 a.m. Horizontal bars indicated the period of time and time of day, when the individual stressors were applied. Specifics of stressors were as follows: smaller cage $(25 \times 20 \times 14 \mathrm{~cm} ; 1 \times 7$ hours, $1 \times 17$ hours); wire cage $\left(1 \times 7\right.$ hours; $1 \times 17$ hours); food and water deprivation $(2 \times 7$ hours $)$; empty water bottle $(1 \times 1$ hour $)$; damp bedding $(1 \times 9$ hours, $1 \times 17$ hours $)$; tilted cage $\left(45^{\circ} ; 1 \times\right.$ 7 hours, $1 \times 17$ hours); crowding ( 8 dams to a standard cage; $2 \times 7$ hours); isolation $(1 \times 17$ hours $)$; new partner $(1 \times 24$ hours $)(1)$

system. The ABR thresholds were measured under general anesthesia (xylocaine solution $(10 \mathrm{mg} / \mathrm{kg}$ ) and ketamine (80 mg/kg) IP) in response to $4,8,12$ and $16 \mathrm{kHz}$ tone burst (5 ms duration, $0.5 \mathrm{~ms}$ rise-fall delivered at 23.1/s) at day 27 or 28. At each SPL, 1024 responses were averaged. Threshold was defined as wave II and was recorded repeatedly. In order to evaluate the ABR latency, click stimulus was delivered at $80 \mathrm{~dB}$ SPL and repetition rate of 11.1 second and rarefaction polarity was implemented.

To analyze the data, SPSS 21 (SPSS Inc, Chicago, Ill, USA) was used and t-test was employed to compare the variable mean values. Differences were considered statistically significant when $\mathrm{P}$ was $<0.5$.

All methods and techniques used in this study were approved by the Ethics Committee of the University of Medical Science with code number 1395.92113.3001.

\section{Results}

The waveforms and general response properties of the ABRs recorded for the present study were all similar to previous studies. The waves mainly consisted of four peaks of P1, P2, P3, and P4. Among all waves, P2 was the strongest and was used to determine the hearing threshold in this study.
The average hearing threshold of ABR was examined at different frequencies between the two groups, which is represented in Figure 2. As seen in this figure, the lowest hearing threshold was related to the frequency of $12 \mathrm{kHz}$ (experimental group: $27.95 \pm 3.1 \mathrm{~dB}$ and control group: $26.73 \pm$ $4.71 \mathrm{~dB}$ ) while the highest hearing threshold was recorded at a frequency of $4 \mathrm{kHz}$ (experimental group: $39.82 \pm 5.37$ $\mathrm{dB}$ and control group: $38.88 \pm 4.71 \mathrm{~dB}$ ). The shape of audiograms was similar. Furthermore, the difference between the two groups in terms of hearing threshold was not significant $(\mathrm{P}>0.05)$.

The wave latency assessment in both groups indicated that ABR waves had higher latencies in the group with a history of prenatal stress exposure. However, the difference between the two groups was statistically significant in P2 (experimental group: $2.40 \pm 0.11 \mathrm{~ms}$, control group: $2.35 \pm$ $0.11 \mathrm{~ms}(\mathrm{P}=0.035)$ ) and $\mathrm{P} 4$ waves (experimental group: 4.39 $\pm 0.23 \mathrm{~ms}$, control group: $4.29 \pm 0.19 \mathrm{~ms}(\mathrm{P}=0.044)$ ).

Evaluation of maternal blood corticosterone levels at the end of pregnancy showed that there was a significant difference in corticosterone of the CMS group $(\mathrm{P}<0.05)$. As indicated in Figure 3, corticosterone level in the CMS group $(151.3 \pm 16.2 \mathrm{ng} / \mathrm{mL})$ was higher than the control group (43.2 $\pm 11.4 \mathrm{ng} / \mathrm{mL}$ ).

Weight evaluation of both groups indicated that rats 


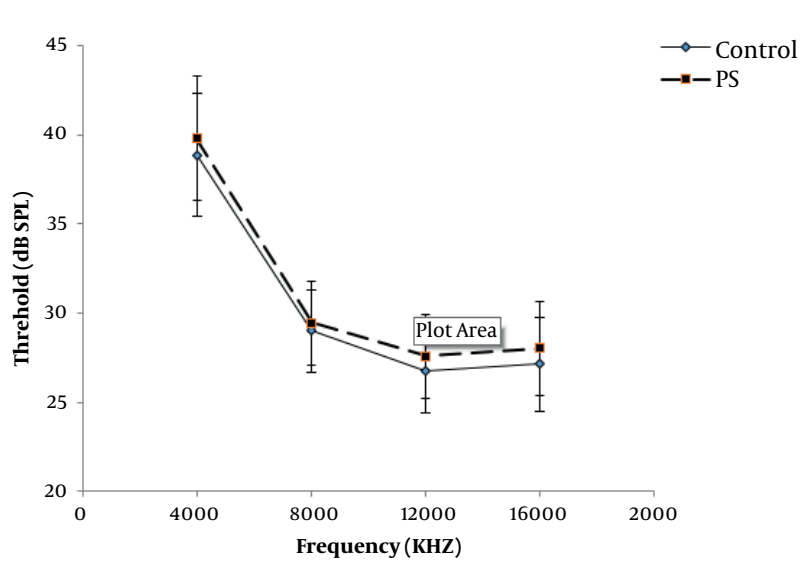

Figure 2. Average hearing threshold of ABR in different frequencies in two groups

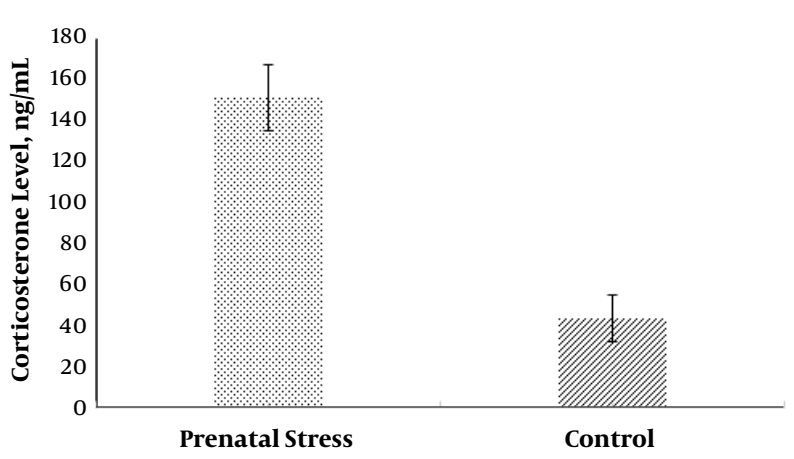

Figure 3. Maternal blood corticosterone levels at the end of pregnancy in prenatal and control groups

exposed to prenatal stress weighted far less than the control group. The average weight of the experimental group (rats exposed to prenatal stress: $57.96 \pm 10.24$ ) was much lower than the control group $(86.03 \pm 20.94 ; \mathrm{P}<0.001)$

\section{Discussion}

This study aimed at determining whether prenatal stress would influence hearing in rat pups. The main finding was that although prenatal stress increased corticosterone level in pregnant rats, it had no effect on hearing threshold of their pups. In addition, prenatal stress caused a significant impact on auditory neural transmission time at the brain stem the pups. It was also found that rats offspring weight was severely reduced, and was affected by stress during pregnancy.

In this study, CMS regimen developed systemic stress in pregnant rats. The chronic mild stressor used in the current experiment was based on those described by other researchers $(1,2)$. While CMS did not yield consistent results across different studies $(16,17)$, the significant increase in corticosterone level suggests that the procedure used in the current experiment was perceived as stressful by the pregnant rats and this was confirmed by several other studies $(18,19)$.

In the present study, ABR was recorded at 4, 8, 12 and $16 \mathrm{kHz}$, and rats' highest auditory sensitivity was seen within the range of 8 to $16 \mathrm{kHz}$. In previous studies, hearing threshold of rats from different strains was examined using both electrophysiological and behavioral methods. In behavioral studies, although rats' hearing threshold was lower than what was recorded in this research (20), the audiogram pattern observed in the present study corresponded with what was reported in previous research (21). In behavioral studies, rats hearing was most sensitive within the range of 8 to $32 \mathrm{kHz}(20)$, and in studies performed using electro-physiological techniques, sensitivity was highest at frequencies of $8(7,21)$ and $16(5) \mathrm{kHz}$. These findings are highly consistent with that recorded in this investigation.

Numerous studies have been carried out focusing on the effect of prenatal stress on the auditory system. In the present article, it was revealed that prenatal stress has no impact on auditory sensitivity. This result is contradictory to results of studies suggesting that stress during pregnancy worsens animals' hearing threshold (5). Nevertheless, most studies, have reported that prenatal stress does not affect hearing threshold $(1,7)$.

Since the effect of stress varies in different individuals (1), in this study, only one offspring was chosen from each under stress pregnant mother to be placed in each group. If more than one offspring rat is chosen from each mother, there will probably be many similarities between data obtained from all groups. This in turn will be a barrier for accurate investigation of the real effect of stress on offspring rats. In their work, Kadner et al. (5) picked more than one offspring rat from each mother, which made statistical first type error more likely. More importantly, the difference between hearing thresholds of the two groups in Kadner et al.'s study was only about $7 \mathrm{~dB}$ and was only found at lower frequencies. Such difference may have been neglected if this point had been taken into consideration when grouping. In contrast, in studies that concentrated on litter effect, it was found that prenatal exposure to stress or injection of dexamethasone during pregnancy did not cause any hearing loss in the offspring $(1,7)$. Another possible reason for difference between results of this study and Kadner et al.'s work (5) is how and in what way the stress protocol is imposed on rats during pregnancy. To implement the experiments, the Willner stress model (2) was used. Whilst, Kadner et al. (5) employed an entirely different stress model. Since the level of stress induced in moth- 
ers can be influenced by the type of stress exposure model (3) and the nature of the stress exposure during pregnancy can alter the function of the HPA axis in the offspring (8), there is a possibility that the protocol used in the study done by Kadner et al. (5) had greater effect on pregnant mothers' HPA axis and auditory system.

Absolute wave latencies assessment was conducted in order to examine the potential effect of prenatal stress on afferent auditory pathways. The results indicated that absolute latency of waves II and IV was increased, which could be a sign that the generators of these waves were more affected. A limited number of studies on the effect of prenatal stress on the auditory system neglected to examine the absolute latency of ABR waves $(1,7)$ yet as stated in previous studies, prenatal stress influences sensory gating (8) and startle reflex (9), thus it is expected to observe some hearing abnormalities at the brain stem of animals with a history of prenatal stress exposure.

To explain the increase in the latency of ABR waves in rats exposed to prenatal stress, several factors can be considered. It was manifested that high concentration of glucocorticoids (GC) can change the biological properties of neurons' plasma membrane, which results in impaired ions movement cycle across the cell membrane and deficiencies of nerve impulse transmission (22). Given that rats exposed to prenatal stress have a higher level of basic GC (5), there is a possibility that their neuronal function is impaired and thus the action potential transmission time is increased (22). Previous research has also reported the malfunction of different parts of the central nervous system (CNS) in animals exposed to prenatal stress (22). During the aging process, similar to prenatal stress exposure, malfunction of HPA axis and rising the basic level of GC in blood can occur (23). Since this process in aging causes disruption of neuronal synapse in CNS and increases the latency of ABR waves (3), it can be expected that exposure to PS would have this outcome as well.

It was shown that prenatal stress led to an increase in latency of waves II and IV while waves I and III were not affected. This could be due to the fact that stress affects various parts of CANS differently (11). It was highlighted in one study that the effect of stress on neuronal atrophy of inferior colliculus (IC) is much more than on the hippocampus (10). It was also revealed that after stress is induced to rats, expression of genes related to glucocorticoid receptor (GR) and Hif-1 (hypoxia-inducible factors) mainly occurs in IC and cochlear nuclei (11). This in indicative of the fact that unlike more peripheral structures, central parts of the afferent auditory pathway are more affected by prenatal stress (10). Since waves II and IV in rats are respectively generated by cochlear nuclei and IC (20), their increased latency time could be caused by greater impact of prenatal stress on their generators.

In this research, the researchers also witnessed lower weight in offspring rats with a prenatal stress experience, which was proved in many previous studies $(1,7,14)$. As Hougaard et al. (1) observed, injection of dexamethasone (a synthetic GC) during pregnancy reduced offspring rats' weight while CMC stress regimen had no such effect. It was noted that many of the defects seen in fetuses under stress were the result of impairment of the HPA axis (14). It seems that HPA axis is considered both a target of peripheral changes and a mediator that links early life events with the health state in adulthood (8). Since HPA axis function is influenced by genetics, in various races (6), and epigenetic factors within the same race (6), differences observed between the results of this study and that of Hougaard et al. (1) could be somewhat caused by such differences. Unfortunately, data associated with fetal and newborns growth has not been addressed in all previous research (5).

\subsection{Conclusion}

The presented results confirmed that prenatal exposure to stress during gestation is not detrimental to hearing sensitivity per se. However, increasing the latency of ABR waves confirmed that higher level of CANS may be affected by prenatal stress. Therefore, processing hearing stimulus in higher levels of hearing afferent pathways may be corrupted.

\section{Acknowledgments}

This research was supported by Iran University of Medical Sciences and this article was part of a PhD thesis.

\section{References}

1. Hougaard KS, Barrenas ML, Kristiansen GB, Lund SP. No evidence for enhanced noise induced hearing loss after prenatal stress or dexamethasone. Neurotoxicol Teratol. 2007;29(6):613-21. doi: 10.1016/j.ntt.2007.07.006. [PubMed: 17804195].

2. Muscat R, Willner P. Suppression of sucrose drinking by chronic mild unpredictable stress: a methodological analysis. Neurosci Biobehav Rev. 1992;16(4):507-17. doi: 10.1016/s0149-7634(05)80192-7.

3. Baker S, Chebli M, Rees S, Lemarec N, Godbout R, Bielajew C. Effects of gestational stress: 1. Evaluation of maternal and juvenile offspring behavior. Brain Res. 2008;1213:98-110. doi:10.1016/j.brainres.2008.03.035. [PubMed: 18456246].

4. Celsi G, Kistner A, Aizman R, Eklof AC, Ceccatelli S, de Santiago A, et al. Prenatal dexamethasone causes oligonephronia, sodium retention, and higher blood pressure in the offspring. Pediatr Res.1998;44(3):31722. doi: 10.1203/00006450-199809000-00009. [PubMed: 9727707].

5. Kadner A, Pressimone VJ, Lally BE, Salm AK, Berrebi AS. Low-frequency hearing loss in prenatally stressed rats. NeuroRep. 2006;17(6):635-8. doi: 10.1097/00001756-200604240-00015. 
6. Ohlemiller KK. Recent findings and emerging questions in cochlear noise injury. Hear Res. 2008;245(1-2):5-17. doi: 10.1016/j.heares.2008.08.007. [PubMed: 18790034]. [PubMed Central: PMC2610263].

7. Canlon B, Erichsen S, Nemlander E, Chen M, Hossain A, Celsi G, et al. Alterations in the intrauterine environment by glucocorticoids modifies the developmental programme of the auditory system. Europ J Neurosc. 2003;17(10):2035-41. doi: 10.1046/j.1460-9568.2003.02641.x.

8. Schneider ML, Moore CF, Gajewski LL, Larson JA, Roberts AD, Converse AK, et al. Sensory processing disorder in a primate model: evidence from a longitudinal study of prenatal alcohol and prenatal stress effects. Child Dev. 2008;79(1):100-13. doi: 10.1111/j.1467-8624.2007.01113.x. [PubMed: 18269511]. [PubMed Central: PMC4226060].

9. Braff DL, Geyer MA, Swerdlow NR. Human studies of prepulse inhibition of startle: normal subjects, patient groups, and pharmacological studies. Psychopharmacol. 2001;156(2-3):234-58. doi: $10.1007 /$ s002130100810.

10. Camarero G, Avendano C, Fernandez-Moreno C, Villar A, Contreras J, de Pablo F, et al. Delayed inner ear maturation and neuronal loss in postnataligf-1-deficient mice. J Neurosci. 2001;21(19):7630-41. doi: 10.1523/jneurosci.21-19-07630.2001.

11. Mazurek B, Haupt H, Joachim R, Klapp BF, Stover T, Szczepek AJ. Stress induces transient auditory hypersensitivity in rats. Hear Res. 2010;259(1-2):55-63. doi: 10.1016/j.heares.2009.10.006. [PubMed: 19840840]

12. Ban JH, Jin SM. A clinical analysis of psychogenic sudden deafness. Otolaryngol Head Neck Surg. 2006;134(6):970-4. doi: 10.1016/j.otohns.2005.11.045. [PubMed: 16730540].

13. Mazurek B, Haupt H, Olze H, Szczepek AJ. Stress and tinnitus-from bedside to bench and back. Front syst neuroscience. 2012;6:47.
14. Barrenas ML, Bratthall A, Dahlgren J. The thrifty phenotype hypothesis and hearing problems. BMJ. 2003;327(7425):1199-200. doi 10.1136/bmj.327.7425.1199. [PubMed: 14630755]. [PubMed Central: PMC274054].

15. Cook CJ. Patterns of weaning and adult response to stress. Physiol Behav.1999;67(5):803-8. doi:10.1016/s0031-9384(99)00107-9.

16. Stout SC, Mortas P, Owens MJ, Nemeroff CB, Moreau J. Increased corticotropin-releasing factor concentrations in the bed nucleus of the stria terminalis of anhedonic rats. Eur J pharmacol. 2000;401(1):39-46.

17. Bielajew C, Konkle ATM, Merali Z. The effects of chronic mild stress on male Sprague-Dawley and Long Evans rats: I. Biochemical and physiological analyses. Behav brain res. 2002;136(2):583-92.

18. Harris RBS, Zhou J, Youngblood BD, Smagin GN, Ryan DH. Failure to change exploration or saccharin preference in rats exposed to chronic mild stress. Physiol Behav.1997;63(1):91-100. doi:10.1016/s00319384(97)00425-3.

19. Wang Y, Liberman MC. Restraint stress and protection from acoustic injury in mice. Hear Res. 2002;165(1-2):96-102.

20. Kelly JB, Masterton B. Auditory sensitivity of the albino rat.JComparat Physiol Psychol.1977;91(4):930-6. doi:10.1037/h0077356.

21. Popelar J, Groh D, Mazelova J, Syka J. Cochlear function in young and adult Fischer 344 rats. Hear Res. 2003;186(1-2):75-84. doi: 10.1016/s03785955(03)00329-0.

22. Jin DX, Lin Z, Lei D, Bao J. The role of glucocorticoids for spiral ganglion neuron survival. Brain res. 2009;1277:3-11.

23. Backoff PM, Caspary DM. Age-related changes in auditory brainstem responses in fischer 344 rats: effects of rate and intensity. Hear Res. 1994;73(2):163-72. doi: 10.1016/0378-5955(94)90231-3. 